THE current number of the British Quarterly Review contains an article on recent Japanese progress, which is by far the most valuable that has been published on this subject for many years past. The anthor, Col. H. S. Palmer, R.E., describes fully the caures and course of the changes which have passed over the "Land of the Rising Sun" in the past fourteen years; the vari us and complicated changes in the constitution and administration - from the pure oligarchy which succeeded the revolution of 1868 , to the system of tolerably free local government of the present day-are clearly explained, and the effect of the various steps in these chanres made comprehensible to the general reader. The writer then takes the recent reforms under various heads - the army, navy, education, public works, prisons, \&c.and shows, by statistics, what advance has really been made. The last half of the paper is, in fact, a comprehensive summary, with running commentary, of the Japanese government statistics in every department. The knotty subject of finance is treated with as much clearness as the subject admits of. Under this head the alinost inevitable character of the present financial depression is explained; but it is gratifying to notice that a careful and impartial observer like Col. Palmer is able to conclude his article with confidence in the future of the country to which he has devoted so much study. Many of the interesting stati-tic; in the paper have already a speared in the columns of NATURE.

Dr. Horub has sent us several papers connected with his Sou'h African explorations. There are two on the English in South Africa, from the standpoint of exploration and civilisation, and a similar paper on the French in Tunis; and an interesting Catalogue, with notes, of Dr. Holub's ethnographical collections.

In connection with Egyptian troubies, Mr. Wyld has published two maps, which may be useful to those who are watching operation:. One is a plan of Alexandria and the harbour, with an inset map showing the British possessions in the Old World; the other is a smalt map of the Isthmu; of Suez and I.ower Egypt, on the scale of twelve miles to an inch, with a similar inset map.

\section{CONTRIBUTION OF ASTRONOMY TO THE PROBLEM OF MOLECULAR PHYSICS ${ }^{1}$}

THE kind way in which you have received me, leads me to fix, by writing, the principal points of our conversation on Sunday last. I thank you heartily for offered help to realise the scientific aim I have in view, and which I will now explain.

The synthetic study of thermo-chemical phenomena, of the laws of thermo-dynamics and of experiments relating to these subjects of the physical sciences, has brought us to consider the temperature of a body as being the mean amplitude of the vibratory oscillation of molecules constituting that body.

This definition, taken as a starting point, enables us to explain and deduce all the essential laws of the mechanical theory of heat. We obtain from it easily the law of Dulong and Petit, that of isomorphism in systems of crystallisation, the relations connecting the coefficients of expansion of all substances with their atomic weight, their temperature of fusion and their density, \&c.

The maximum tensions are calculated in advance with all exactness, and lastly, the two great mechanical principles of heat are an immediate and necessary consequence of it.

I have, then, every reason to believe that this definition will be adopted, since it satisfies as well the condition of integrability of the differential equation of motion (function S of Zeuner) as the definition drawn from the air or mercury thermometer (definition of Regnault).

In that case, what is the specific heat of a body?

The specific heat becomes the sole manifestation of the atiraction of molecules for one another.

Indeed, if we $\mathrm{n}$ ultiply the space traversed (temperature) by the molecular force (specific heat), we obtain the total heat or quantity of absolute work which the substance contains.

Here, consequently, comes in an important question, which is by no means secondary, as has often been said-

Is the attraction of matter for matter a fundamental essential property of matter, or is it merely the result of the dynamical action of the medium in which the matter exists?

In other terms, may one say, without its being possible to ex-

I A letter from M. Raoul Pictet to $M$. Dumas, dated Paris, December $x 6$, 1881, and published in Archives des Sciences, June 15. plain it, Matter attracts matter without the active intermediary of the medium; or, Attraction as a force does not exist; it is merely the manifestation of shocks of the ether which tend to approximate bodies according to the Newtonian law ?

In the former case, one regards the attractive potential of matter as an original capital place in each material element, a capital which is only exhausted by the absolute approximation of all matter existing in the universe. In the latter case this potential is nil, and one supposes that a certain quantity of kinetic enersy has been communicated in the beginning of time to the mass of the universe, a quantity of energy which is inevitably transformed under a thousand different combinations into all the physico-chemical and astronomical phenomena of nature.

In the former case $\frac{1}{2} m y^{2}+$ the potential is constant.

In the latter, $\frac{1}{2} m \gamma^{2}$ alone is constant. The solution of this important question i; necessary to establi $\rightarrow$ h physical theories in a somewhat distinct manner, and to prove the intimate relation existing between the various elements of bodies.

$\mathrm{On}$ the hypotbesis that attraction is an essential property of matter, we shall liken it to inertia; thus any body will possess as primordial characters, a certain quantity of inertia, without which we should never come to be put in contact with it nor to know it, and a certain quantity of attraction, which will be the manifestation of its proper influence on the rest of the universe. Such will be the conditions of existence of matter.

On the hypotheses that $\frac{1}{2} m v^{2}$ alone is constant, inertia and motion are the fund imental properties of matter; shocks are the means of transformation of different modes of motion.

Let us take, then, any body and heat it.

If we are partisans of the first hypothesis, that of potential, we must expect to find simple relations between the inertia of the body considered, the attraction of the molecule for one another, and increase of volume of the body, the whole associated with the quantity of mechanical work furnished to the body in the form of heat.

The specific heats and latent heats will then be functions of the atomic weight or inertia of the body, and the dissociation which is manifested by fusion and volatilisation, will be deduced from the study of the body under these two aspects, masses set in motion, and potential of those masses.

If we are partisans of the second hypothesis, supposing that $\frac{1}{2} m v^{2}$ is constant, we are obliged simply to consider the volume of the body ; that is to say, the exterior surface of the smallest quantity of matter.

Indeed, shocks alone explain the phenomena. But when one says shock, he says surface where the shock occurs. The greater the surface the larger tha number of shocks of the ether, the stronger the reaction of the matter.

We must expect them, in this second hypothesis, to find simple relations between the volumes of atoms and of molecules, that is, between the co-efficients which represent the density of the bodies, the number of atoms and the atomic weight, and the specific heats, latent heats, and maximum tensions.

In other terms, in the first hypothesis, molecular physics will rest essentially on the atomic wight, which, by virtue of the fall of bodies, represents simultaneously the idea of inertia and that of attraction, essential properties; in the second hypothesis the physico-chemical phenomena are deduced mainly from the volume of atoms and the medium in which the phenomena occur.

The medium becoming active, a variation of medium will induce-in phenomena of attraction concomitant variations quite independent of matter itself.

The specific heats and the latent heats may then be variable elements in the same substance and at the same temperature under the same pressure, according to the mechanical energy of the medium in which the phenomena occur.

Thus the whole of molecular physics is closely connected with the solution of this theoretical question.

We have sought an experimental method capable of throwing some light on this problem. Without entering into too minute details, we will explain the plan of this work.

It may be accepted, I believe, that the solar system is nearly independent, mechanically speaking, of the rest of the universe ; that is to say that no motion, relatively to the centre of gravity of this system, is produced in our planets by the perturbation of other systems surrounding us.

We niay then call $M$ the total mass of the solar system. This mass is decomposed into $m, m^{1}, m^{2}$. . the respective masses of the Sun, Venus, the Earth, Jupiter, $\dot{\&}$., and $\mu$ the mass of 
the ether, whose density is function of the velocity of propagation of light and heat, as also of the wave-lengths.

Multiplying all these masses by the square of the velocity of each particle relatively to the centre of gravity of the solar system, we obtain the factor $\frac{1}{2} \mathrm{M} v^{2}=$ the total kinetic energy of the solar system.

This constant kinetic energy (if the second hypothesis be admitted, in which $\frac{1}{2} m v^{2}$ is constant) is not distributed throughout the solar system in a regular and fixed manner. Sometimes a planet, as Jupiter, is at the extremity of the larger axis of his ellipse, and advances more slowly; sometimes, on the contrary, his velocity is accelerated and passes through a maximum to another position of his orbit.

At the same minute all the planets are revolving round the sun, some with their maximum velocity, others with their minimum velocity, others, again, with intermediate velocities. We may make addition of all these kinetic energies of the whole solar system, and differentiate the total equation with reference to time. The variations thus obtained for each hour will naturally eliminate all the quantities of constant kinetic energy represented by the rotaticn of the stars on their own axes ; they will merely show the increase or the diminution of the whole of the variable kinetic energies of the svstem.

One may easily draw a curve of these variations calculated by the ephemerides of the principal planets. Jupiter will play a preponderating rôle in this calculation.

Considering still the second hypothesis, in which the attraction is merely the result of shocks, it is evident that the attraction manifested by each planet for the bodies which are on its surface will be the echo of the kinetic energy disposable on this planet. This kinetic energy will be variable according to the day and hour of observation.

In fact, the kinetic energy of the solar system being fixed and constant, if the planets, on a certain day, absorb into their own mass a maximum quantity of kinetic energy, the cause of gravity on the earth will be diminished by the whole of the excess which is accumulated in these bodies in motion, and the acceleration $g$ will pass through a minimum. On the other hand, when, a few years later, the whole of the planets give a minimum total of kinetic energy for their masses in motion, the value of $g$, for the same reasons, must pass through a maximum.

It is easily understood that the value of the terrestrial attraction cannot remain con tant if the disposable kinetic energy varies in function of the time and of the respective position of the other planets.

Now, we may calculate the total mass $M$ of the system, the partial masses and their variable velocities; we obtain for these variations considerable values; then if we register carefully the values of $g$ obtained directly. during observations which must continue at the least several years, and if we trace a curve of the values of $g$ so obtained, we should find the following coincidence :-

The curve of variations of the total kinetic energy of the planets must be inverse to the curve of values of $g$ referred to the same time.

The differences between the maxima and the minima of the two curves, taken on the same ordinate, will give the measure of the velocity of propagation of the kinetic energy in the ether of the solar system.

These conclusions are rigorous in the case of the hypothesis,

$$
\frac{1}{2} m v^{2}=\text { constant, }
$$

being in accurdance with nature.

In the case, on the other hand, of attraction being an essential property of matter, and of our having-

$$
\frac{1}{2} m v^{2}+\text { the potential }=\text { constant, }
$$

we should find for $g$ a constant, since $g$ is the sole manifestation of a constant potential, supposing the mass of the earth is constant during the course of the observations of $g$.

It will be necessary, then, to take account of perturbations of the moon for the measurements of $g$, as also of those of the sun, then to verify whether, these corrections having been made, $g$ is constant.

I believe that this experimental method is the only means we possess of diagnosticating with certainty on the essential properties of matter, and of deciding between those two great theories which are both maintained by men of incontestable merit.

As to the measurement of $g$, there are several oferative processes, and it will be indispensable, before commencing observations, to discuss analytically the advantages of each of them, and the modes of inscription of the values obtained.
The optical means of registration, the mechanical actions connected with the motion of pendulums, and the kind of pendulums, will be so many important subjects of discussion, in the case of taking these researches in hand, which I consider as very useful for the definitive settlement of physical theories.

This is a rather long letter, you see, dear Teacher; but I thought to explain to you the object which I pursue, in its general traits, happy indeed if the experiments may be undertaken under your benevolent auspices.

Accept, dear Teacher, I pray you, the expression of my gratitude and entire devotion. Raoul Pictet

\section{A GEOMETRICAL CONSTRUCTION GIVING THE RELATTON BETWEEN THE WASTE AND USEFUL WORK IN A SHUNT DYNAMO}

THE ratio between the portion of electrical energy utilisable in the external circuit of a shunt dynamo and the portion wasted in heating the wire of the armature and field magnet is easily calculated as soon as one knows the resistances of the armature and magnet wires, and the resistance equivalent to the external circuit; and I do not know that there is any great advantage in putting it into a geometrical form. Still there are people who prefer a cunstruction to a formula, and the following construction is easily made, especially with the use of squared paper.

In the figure annexed, let o $\mathrm{A}$ represent the resistance of the armature between the points where the branching occurs; o $B$

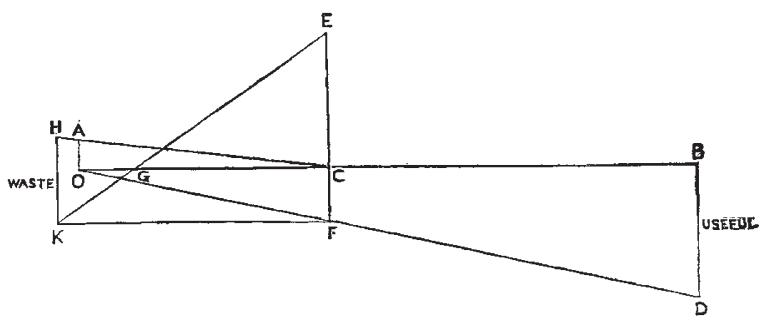

the resistance of the field magnet wire; and $\mathrm{OC}$ the resistance of the external circuit, or its equivalent.

Erect lines to represent the useful work (E.M.F. $\times$ current), on any convenient scale, at $\mathrm{C}$ and at $\mathrm{B}$; viz. $\mathrm{C} E$ and $\mathrm{B} D$.

Join $\mathrm{O} D$, producing $\mathrm{EC}$ to meet it at $\mathrm{F}$.

Lay off C G equal to E F ; draw E G and a horizontal through F. Then from their meeting point $\mathrm{K}$ draw a vertical, meeting $\mathrm{C} A$ in $\mathrm{H}$.

The length $\mathrm{HK}$ so determined represents the waste portion of the total electrical energy, on the same scale as $B D$ or E C represents the useful.

In this figure the effect of the armature resistance in tilting up the line $\mathrm{CA}$ and so increasing the waste is very manifest; the increase of waste by decreasing the resistance $O B$ is somewhat less striking, but quite distinct ; the effect of a change in $\mathrm{OC}$ is, as it should be, not so obvious. It may be noted that the most economical value for $\mathrm{OC}$ is very nearly indeed a geometric mean between $\mathrm{OA}$ and $\mathrm{OB}-\mathrm{OA}$; which is an easy rule to apply in practice.

Liverpool, July I9

OLIVER J. LODge

\section{UNIVERSITY AND EDUCATIONAL INTELLIGENCE}

THE following is the list of candidates successful in the competition for the Whitworth scholarships, 1882, in connection with the Science and Art Department :-Charles Webster, apprentice; John H. Tomlinson, apprentice ; James M. Beaman, fitter; Thomas Turner, engineer; D. Codrington Selman, engineer; Charles B. Outon, draughtsman; George $H$. Banister, draughtsman; Frederick Lane, fitter; William D. Laird, engine fitter; Joseph Parry, engine fitter; Albert F. Ravenshear, apprentice; Charles W. Carter, brass-finisher; Alfred Barrow, fitter ; Henry C. King, fitter ; Malcolm Douglas, apprentice ; Thomas H. Gardner, engineer ; Ernest E. Haine, engineer; George Halliday, engineer; George W. Buckwell, draughtsman ; Louis H. Cochrane, engineer; William Duncanson, engine fitter; Henry Brown, engineer; William T. Hatch, 